\title{
IPTV Delivery Architecture in 10G EPONs using ONU-Based Multicast Emulation
}

\author{
Su-il Choi* \\ School of Electronics and Computer Engineering, Chonnam National University \\ 300 Yongbong-dong, Buk-gu, Gwangju, 500-757, Republic of Korea
}

(Received June 13, 2008 : accepted June 17, 2008)

\begin{abstract}
EPONs are a low cost, high speed solution to the bottleneck problem of broadband access networks. To support point-to-point and shared LAN emulation, EPONs use the multi-point control protocol (MPCP), which uses logical link identification (LLID) forframe tagging and filtering between the OLT and ONUs. In this paper, ONU-based multicast or multiple shared LAN emulation for IPTV services is proposed using logical group identification (LGID). Using ONUbased VLAN services, EPONs can support separate and secure connections between providers and subscribers in a simple manner. Also, differentiated IPTV channel packages can be delivered through EPONs by implementing ONU-based VLAN and IGMP snooping mechanisms.
\end{abstract}

Keyword: EPONs, LLID, LGID, MPCP, point-to-point emulation, shared LAN emulation, multicast, IPTV

OCIS codes : (060.4250) Networks; (060.4230) Multiplexing; (060.0060) Fiber optics and optical communications; (060.4510) Optical communications

\section{INTRODUCTION}

Access networks connect business and residential subscribers to the central offices of service providers, which in turn are connected to metropolitan area networks (MANs) or wide area networks (WANs). Recently, Ethernet PONs (EPONs) have attracted a great deal of interest in both industry and academia as a promising cost-effective solution for next-generation broadband access networks. As an inexpensive, simple, and scalable technology, and with the capability of delivering integrated services, EPONs, whose aim is to significantly increase the broadband service performance while minimizing equipment, operation, and maintenance costs, are standardized in the IEEE 802.3ah version of Ethernet [1]. EPONs consist fundamentally of an optical line terminal (OLT) located at the central office (or cable headend), and multiple remote optical network units (ONUs) that deliver broadband voice, data and video services to subscribers. The only interior elements used in a passive optical network are passive optical components, such as optical fiber, splices, and

${ }^{*}$ Corresponding author: sichoi@email.jnu.ac.kr splitters.

In the downstream direction, the OLT broadcasts frames and the ONUs selectively receive those frames addressed to themselves. The broadcasting nature of Ethernet is perfectively suited to the downstream transmission mechanism of EPON. In the upstream direction, each ONU's transmissions are received by the OLT, but not by any other ONU. Therefore, an EPON, which is referred to as a point-to-multipoint network, operates in reality as a point-to-multipoint network in the downstream direction and a point-topoint network in the upstream direction. However, the IEEE 802.1D standard, which specifies the operation of a bridge, takes only two LAN types, the point-to-point local area network (LAN) and shared LAN, into account [2]. Hence, the multi-point control protocol (MPCP) is introduced to provide support for point-topoint emulation and shared LAN emulation in EPONs. Using the MPCP, ONUs selectively receive those frames destined for themselves by matching the logical link identification (LLID) field in the Ethernet frame preamble. In the upstream direction, the ONUs should share the channel capacity and resources, and the buffered frames are "bursted" out to the OLT in the exclusively 
assigned timeslot at the full channel speed [3-6].

Link segregation in access networks is another important problem for service providers and subscribers. The protection of contents for the service provider and data privacy for the subscriber are indispensable to ensure secure service and billing. In EPONs, ONU-based VLAN services would be a simple solution for link segregation. N. Finn [7] proposed the broadcast group identification to support the emulation of multiple shared LANs, but it is only applicable to multicast in the downstream direction. Therefore, in this paper, logical-group identification (LGID) is proposed for ONU-based multicast or multiple shared LAN emulation in EPONs.

Streaming digital video over IP core and access platforms, with various networking mechanisms in place to ensure QoS, has come to be called "IPTV." In an access network, increased quality of service (QoS) is necessary to deliver some services; for example, a multichannel television service offering programs similar to those of today's broadcast or cable television, where the response to changes in the channel is nearly instantaneous and the contents are always available [8]. As of now, 10G EPON technology, which is in the process of standardization by the IEEE P802.3av 10GEPON task force, is suitable for handling multimedia-based bidirectional communication services. Figure 1 shows a 10G asymmetric EPON system which is suitable for bandwidthintensive services such as IPTV. In point-to-multipoint access networks, ONU-based multicast emulation could be used to provide IPTV services with an adequate level of QoS. A possible architecture for digital television over EPON is proposed in [9]. The statistical multiplexing on the Ethernet layer is performed by the IGMP snooping mechanism. However, the multicast frame is broadcasted from the OLT to all ONUs. J. Wu et al. [10] presented a controllable multicast architecture for IPTV services over EPON. OLT assigns a multicast LLID (MLLID), which is described in [11], for the delivery of the multicast stream, so as to save bandwidth resources. However, this is not suitable for IPTV

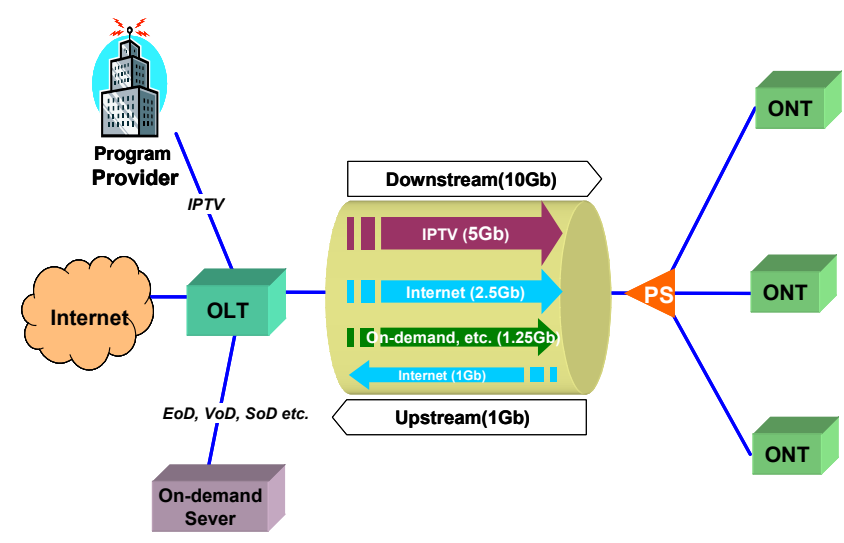

FIG. 1. 10G asymmetric EPON. channel package delivery services over EPONs. By implementing ONU-based VLAN and the IGMP snooping mechanism, 10G EPONs can support separate and secure connections between providers and subscribers, and provide subscribers with differentiated IPTV channel packages, while providing an improved channel zapping time.

Section II introduces the MPCP protocol for EPONs and describes the LLID for point-to-point emulation and shared LAN emulation. Section III presents the proposed LGID, preamble format, frame tagging and filtering rules for ONU-based VLAN services. In Section IV, ONU-based VLAN and the IGMP snooping mechanism are utilized in 10G EPONs to support IPTV channel package delivery services with faster channel switching. Finally, some concluding remarks are given in Section V.

\section{MULTI-POINT CONTROL PROTOCOL}

By defining the multi-point MAC control sublayer as an extension of the MAC control sublayer, the multipoint control protocol can be used to operate an optical multi-point network. The MPCP specifies the control mechanism between the OLT and ONUs connected to a point-to-multipoint segment to allow the efficient transmission of data. The MPCP uses messages, state machines, and timers to control the access to a pointto-multipoint topology. The control messages included in the MPCP are GATE, REPORT, REGISTER REQ, REGISTER, and REGISTER_ACK. Each ONU in the point-to-multipoint topology contains an instance of the MPCP protocol, which communicates with an instance of the MPCP in the OLT. Some of the objectives of the MPCP are to provide support for point-to-point emulation, multiple LLIDs and MAC clients at the OLT, a single LLID per ONU and a mechanism for single copy broadcasting.

The MPCP has two operation modes: normal and auto-discovery. In normal mode, the MPCP relies on two Ethernet control messages, GATE and REPORT, to allocate bandwidth to each ONU. The GATE message is used by the OLT to allocate an upstream transmission window to an ONU. The REPORT message is used by an ONU to report its local conditions to the OLT. Note that the MPCP does not specify any particular DBA algorithm, but simply provides a framework for the implementation of various DBA algorithms [3-6]. In auto-discovery mode, the protocol relies on three control messages, REGISTER, REGISTER_REQ, and REGISTER_ACK, which are used to discover and register a newly connected ONU and collect relevant information about it, such as its round-trip delay and MAC address. The OLT performs registration by assigning a unique logical link identification to the newly connected ONU. 


\section{Logical Link Identification}

The reconciliation sublayer of EPONs is extended to enable multiple data link layers to interface with a single physical layer. The logical link identification is defined as a numeric identifier assigned to a point-tomultipoint association between the OLT and an ONU established through the point-to-point emulation sublayer. This results in the assignment of a value to the LLID variable associated with a MAC, after the exchange of discovery handshake messages between the OLT and ONU. The point-to-multipoint association, which is assigned a unique LLID, is bound to an ONU DTE.

By combining point-to-point emulation, suitable filtering rules at the ONU, and suitable forwarding/reflecting rules at the OLT, it is possible to emulate a shared LAN. This is performed in a layer above the MAC that behaves like an n-port bridge while deciding whether to forward a frame. Also, the mode variable is used to differentiate between unicast frames and broadcast frames.

At the OLT, the rules for setting the mode and LLID parameters are as follows:

- If the OLT sends a unicast frame passed down from the upper layers to an ONU, then it does so with the LLID variable set to the value of the destination ONU and the mode bit set to zero.

- If the OLT sends a broadcast or unknown frame passed down from the upper layers to all ONUs, then it does so with the LLID variable set to the value of broadcast and the mode bit set to one.

- If the OLT sends a unicast frame received from an ONU to another ONU, then it does so with the LLID variable set to the value of the destination $\mathrm{ONU}$ and the mode bit set to zero.

- If the OLT sends a broadcast or unknown frame from an ONU to other ONUs, then it does so with the LLID variable set to the value of the source ONU and the mode bit set to one.

If the OLT receives frames from the ONUs, the rules for filtering are as follows:

- The received mode bit is ignored.

- If the received LLID value matches the value of broadcast and an enabled MAC exists whose LLID variable has the same value, then the comparison is considered a match to this MAC.

- If the received LLID value is any value other than the value of broadcast and an enabled MAC exists whose LLID variable matches the received LLID value, then the comparison is considered a match to this MAC.

- If no match is found, then the frame is discarded within the reconciliation sublayer. If a match is found, then the frame is intended to be transferred. If theframe is transferred, both octets of the LLID

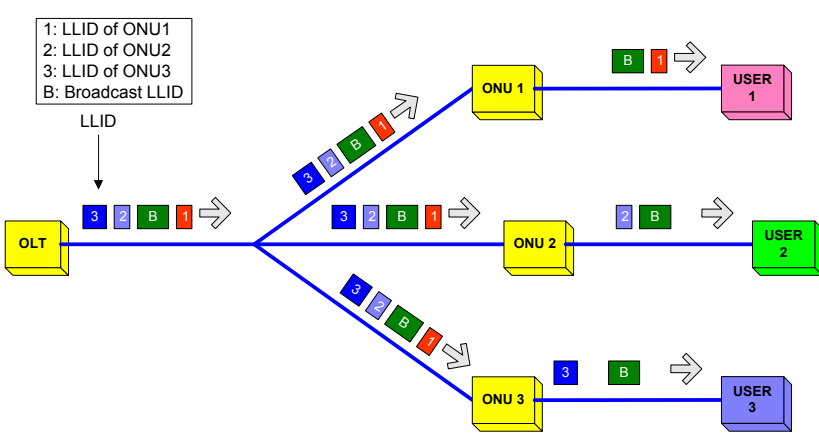

FIG. 2. Point-to-point emulation and single copy broadcast emulation.

field are replaced with normal preamble octets.

When an ONU sends frames to the OLT, it does so with the corresponding LLID and mode bit set to zero. Also, if there are incoming frames from the OLT, the filtering rules of these frames are as follows:

- If the received mode bit is set to zero and the received LLID value matches the LLID variable then the comparison is considered a match.

- If the received mode bit is set to one and the received LLID value doesn't match the LLID variable, or the received LLID matches the value of broadcast, then the comparison is considered a match.

- If no match is found, then the frame is discarded within the reconciliation sublayer. If a match is found, then the frame is intended to be transferred. If the frame is transferred, then both octets of the LLID field are replaced with normal preamble octets.

Figure 2 shows the point-to-point emulation and the single copy broadcast emulation. The unicast frames, transmitted from the OLT, are received by the MAC of the associated ONU DTE. The broadcast frames, transmitted by the OLT, are received by all ONUs.

\section{ONU-BASED VLAN SERCICES}

EPONs can support separate and secure connections between providers and subscribers using ONU-based VLAN services. To support the emulation of multiple shared LANs in EPONs, logical-group identification is proposed to identify a subset of the ONUs that are connected to the same OLT. Also, new frame tagging and filtering rules are proposed for the OLT and ONUs. ONU-based VLAN services would be a simple solution for link segregation that would provide service providers with content protection and subscribers with data privacy.

\section{Logical Group Identification}

A logical group is defined as a set of logical links and 


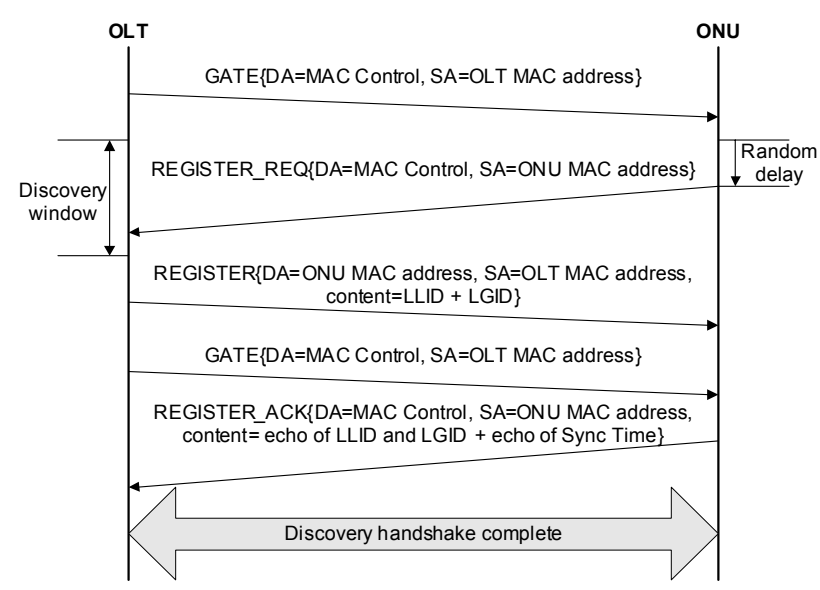

FIG. 3. Discovery handshake message exchange.

\begin{tabular}{|c|c|c|c|c|c|}
\hline & $\begin{array}{r}\text { SL } \\
0 \mathrm{xd}\end{array}$ & 25 & & & \begin{tabular}{c|c} 
LLID \\
$(10 \mathrm{bit})$
\end{tabular} \\
\hline
\end{tabular}

FIG. 4. Suggested EPON preamble format

is identified by a logical group identifier. The LGIDs for an ONU can be assigned during discovery processing and can be changed during normal operation mode. The proposed discovery handshake message exchange is illustrated in Figure 3. After the exchange of discovery handshake messages between the OLT and ONU, it results in the assignment of values to the LLID and the LGID variables associated with a MAC.

The reconciliation sublayer of EPONs is extended to enable multiple shared LANs to interface with a single physical layer. There is a mapping between the sets of \{mode, LLID, LGID\} and multiple MACs. This mapping is used to replace the transmitted preambles with the mode, LLID and LGID fields, as well as to steer the received packet to the appropriate MAC.

Figure 4 shows the suggested Ethernet preamble format for EPONs. The start of the LLID delimiter (SLD) field is used to locate the MODE, LLID, LGID, and 8-bit cyclic redundancy check value (CRC8) fields. The last two octets of the Ethernet preamble are replaced by the MODE, LLID and LGID fields. Some fields of the EPON preamble are as follows:

- MODE: This variable is set to zero for an ONU MAC and may be zero or one for an OLT MAC. When the LLID is used to emulate a single copy broadcast or multicast channel, this variable is set to one. When emulating a unicast channel, this variable is set to zero.

- LGID: This variable may be set to any value for a group of enabled OLT MACs and registered ONU MACs.

- LLID: This variable is set to the broadcast value of 0x3ff for an unregistered ONU MAC. Enabled OLT MACs may use any value for this variable. Registered ONU MACs may use any value other
TABLE 1. Suggested frame reception rules

\begin{tabular}{|c|c|c|c|c|}
\hline \multicolumn{3}{|c|}{ Frame Tag } & \multicolumn{2}{|c|}{ Receive Action } \\
\hline MODE & LGID & LLID & $\begin{array}{l}\text { OLT to } \\
\text { ONU }\end{array}$ & $\begin{array}{l}\text { ONU to } \\
\text { OLT }\end{array}$ \\
\hline \multirow{4}{*}{0} & \multirow{3}{*}{ match } & Match & accept & \multirow{2}{*}{ accept } \\
\hline & & 0x3FF & discard & \\
\hline & & no match & discard & discard \\
\hline & no match & $\begin{array}{c}\text { match/ } \\
\text { no match }\end{array}$ & discard & discard \\
\hline \multirow{3}{*}{1} & \multirow[b]{2}{*}{ match } & match & discard & \multirow{3}{*}{ discard } \\
\hline & & $\begin{array}{c}\text { no match/ } \\
\text { 0x3FF }\end{array}$ & accept & \\
\hline & no match & $\begin{array}{c}\text { match/ } \\
\text { no match }\end{array}$ & discard & \\
\hline
\end{tabular}

than 0x3ff for this variable.

To emulate unicast and multicast channels, the frame reception rules at the OLT and the ONUs are listed in Table 1. At the OLT, the rules for setting the mode and LLID parameters for each shared LAN defined by the LGID variable are as follows:

- If the OLT sends a unicast frame that is passed down from the upper layers to an ONU, then it does so with the LGID variable set to the value of the associated shared LAN, the LLID variable set to the values of the destination ONU, and the mode bit set to zero.

- If the OLT sends a broadcast or unknown frame that is passed down from the upper layers to ONUs, then it does so with the LGID variable set to the value of the associated shared LAN, the LLID variable set to the value of broadcast, and the mode bit set to one.

- If the OLT sends a unicast frame received from an ONU to another ONU, then it does so with the LGID variable set to the value of the associated shared LAN, the LLID variable set to the value of the destination ONU, and the mode bit set to zero.

- If the OLT sends a broadcast or unknown frame from an ONU to other ONUs, then it does so with the LGID variable set to the value of the associated shared LAN, the LLID variable set to the value of the source ONU, and the mode bit set to one.

If the OLT receives frames from the ONUs, the filtering rules are as follows:

- The received mode bit is ignored.

- If the received LLID value matches the value of broadcast (0x3ff) and an enabled MAC exists whose LGID and LLID variables match the received LGID value (0x0) and LLID value, respectively, then the comparison is considered a match to this MAC. 


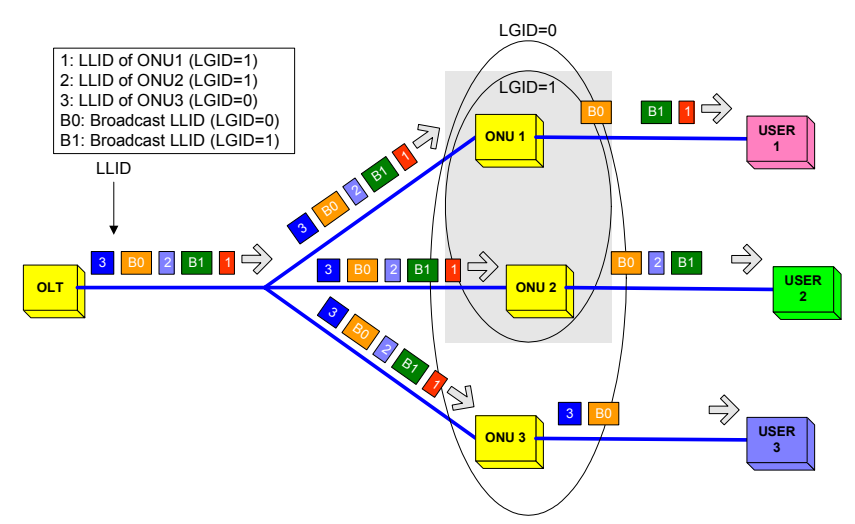

FIG. 5. Point-to-point emulation and multicast channel emulation.

- If the received LLID value is any value other than the value of broadcast and an enabled MAC exists whose mode variable has a value of zero and LGID and LLID variables match the received LGID and LLID values, respectively, then the comparison is considered a match to this MAC.

- If no match is found, then the packet is discarded within the reconciliation sublayer. If a match is found, then the packet is intended to be transferred. If the packet is transferred, then both octets of the MODE, LGID and LLID fields are replaced with normal preamble octets.

When an ONU sends frames to the OLT, it does so with the corresponding LGID and LLID values and mode bit set to zero. Also, if there are incoming frames from the OLT, the filtering rules applied to these frames are as follows:

- If the received mode bit is zero and the received LGID and LLID values match the LGID and LLID variables, respectively, then the comparison is considered a match.

- If the received mode bit is one, the received LGID value matches the LGID variable, and the received LLID value doesn't match the LLID variable, or the received LLID matches the value of broadcast, then the comparison is considered a match.

- If no match is found, then the packet is discarded within the reconciliation sublayer. If a match is found, then the packet is intended to be transferred. If the packet is transferred, then both octets of the MODE, LGID and LLID fields are replaced with normal preamble octets.

Using the proposed multiple shared LAN emulation, ONU-based VLAN services, configured to have separate MAC address databases, can provide each group of subscribers with secure data, voice, and video traffic transmissions. Figure 5 shows a point-to-point emulation and multicast channel emulation. Unicast frames, trans- mitted from the OLT to ONUs, are transferred to the MAC of the associated ONU DTE, and multicast or broadcast frames for a specific group of ONUs are transferred to the MACs of the associated ONUs.

\section{IPTV SERVCES IN 10G EPONS}

Contemporary TV technology sends all TV channels to all end users and channel switching is performed by simple filtering of the undesired frequencies. This mechanism is used in existing cable TV systems. Specifically, changing channels is accomplished simply by selecting one channel and ignoring the rest using a process similar to a band pass filter. However, IPTV cannot make use of this approach, because of the limited bandwidth reaching the home. Therefore, the telecom service provider only sends the selected TV channel or channels to the customer premises. If IP multicast technology is used to deliver IPTV, multicast trees should first be established with QoS guarantees for video transmission.

Conventionally, each user joins a multicast group separately and receives an individual copy of each multicast data packet. When the subscriber sends in a request for a video channel using the STB connected to the $\mathrm{CPE}$, the carrier Ethernet filters the request and replicates and forwards only the requested channel to the subscriber. In an EPON access network, it is inefficient to deliver a separate copy of the packet to each member of the multicast group. One way to improve the efficiency and utilization of an EPON is to take advantage of its broadcast nature when delivering internet group management protocol (IGMP) multicast packets.

\section{Multicast of IPTV channel using LGID}

Fiber to the home (FTTH) is being considered for the deployment of EPONs. In this topology, the ONU is connected directly to an end-user. In an EPON, multiple ONUs may join an IGMP multicast group to receive multicast data, such as video. The OLT processes and responds to the IGMP messages sent by a user, thereby functioning as an IGMP proxy. The OLT communicates on behalf of all the members of a particular multicast group with the multicast source, and receives only one copy of the multicast data. In general, after receiving a multicast data packet, the OLT broadcasts it to all ONUs within the EPON by attaching the broadcast LLID value to the preamble of the Ethernet frame. An ONU which is a member of the multicast group extracts the multicast packet from the Ethernet layer and forwards it to the user.

To implement multicast technology between the OLT and ONUs, we applied ONU-based multicast emulation using LGID. The registration and subscription procedures are shown in Figure 6. During the registration 


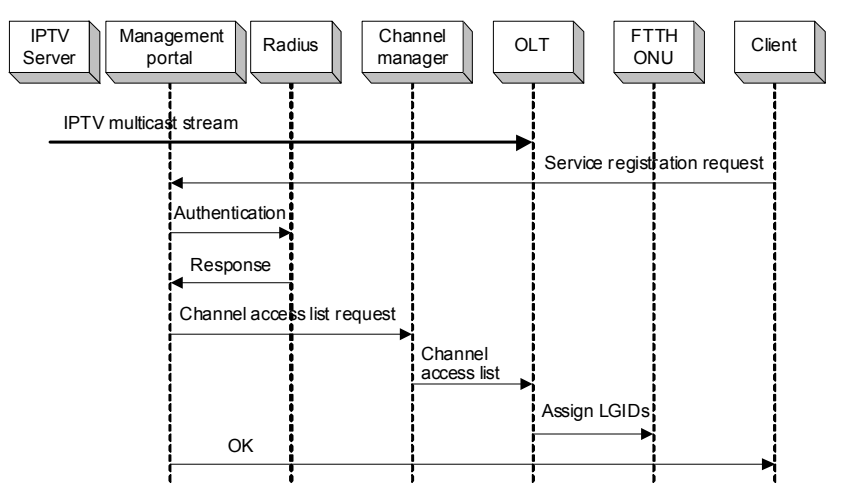

(a) Registration procedure

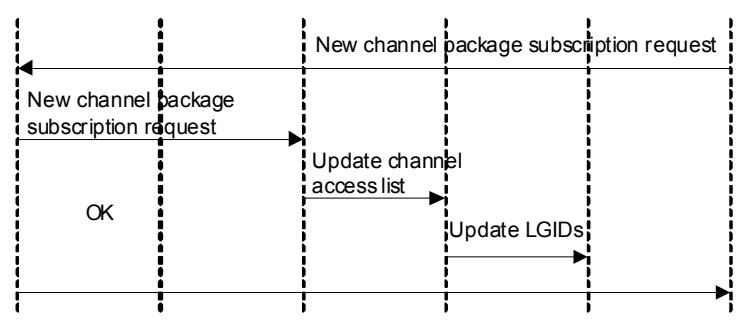

(b) Subscription procedure

FIG. 6. Point-to-point emulation and multicast channel emulation.

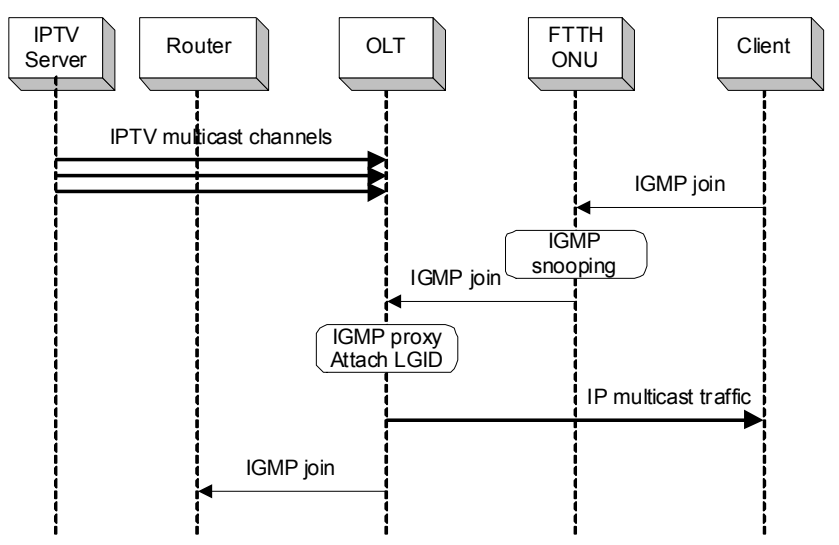

FIG. 7. IPTV delivery using LGID.

procedure, the user sends a service registration request to the management portal. Then, after the user passes the authentication stage, the channel manager sends the channel access list of this user to the OLT and the OLT assigns LGIDs for each ONU. In order to implement channel switching, every IPTV program is previously allocated an IP multicast address and a corresponding multicast MAC address. After registration, when he or she wants to subscribe to a new IPTV channel or channel package, the user sends a subscription request to the channel manager through the management portal. After the channel manager confirms that this request can be accepted, it updates the corre- sponding channel list and sends the updated version to the OLT. The unsubscription procedure can be similarly described. The management of the channel access list of each ONU is performed in the OLT. Thus, the management of the channel access lists is a centralized process.

We assume that IPTV channel packages are delivered to the OLT when an associated channel request is received from any ONU. The IPTV channel delivery procedure is shown in Figure 7. Firstly, the user sends an IGMP request to the ONU. Then, the ONU performs the IGMP snooping function, which is responsible for listening to the IGMP message from the user and setting up a multicast mapping table between the multicast address and the requesting port. The ONU forwards the IGMP request to the OLT which then starts up an IGMP proxy to judge whether to forward the request to the multicast router or not. Because the requested channel already exists, it will drop the IGMP request, and attach the assigned LGID to the IPTV multicast stream and deliver it to the ONUs. The OLT maintains a mapping table between the multicast address and ONUs, which can be used to tell the OLT which ONUs the multicast stream should be forwarded to. After receiving a multicast data packet, the OLT attaches a unique LGID for each multicast address, and delivers it to the allowed ONUs. Then, an ONU which is a member of the multicast group extracts the multicast packet by checking the LGID value of the Ethernet frame, and forwards it to the user.

\section{Multicast of IPTV channel package using LGID}

When it is used in the network, IP multicast technology offers interesting challenges in terms of capacity optimization. While little statistical multiplexing occurs for constant bit rate TV traffic, the key factor that affects the capacity utilization is the packaging of the channels into different IP multicast groups [9]. At one end of the spectrum, each TV channel could be allocated its own IP multicast tree, thus potentially allowing for the bandwidth-efficient delivery of only the requested channels to different branches of this tree. Such a solution would typically result in higher management overhead and increased channel change latency. At the other extreme, the entire set of broadcast channels could be packaged into a single IP multicast tree. Multiple multicast trees allow for fine-grained traffic engineering, while smaller numbers of trees carrying multiple programs would reduce the amount of signaling and other overhead. IPTV support systems must provide for the systematic evaluation of subscriber and channel profiles, in order to identify the most effective channel packaging solutions.

Cable TV providers typically provide market segmentation via subscriptions to different program packages. Similarly, it is expected that IPTV customers will sub- 
TABLE. 2. Management of differentiated IPTV channel package

(a) IPTV channel package table

\begin{tabular}{c|c|c|c}
\hline $\begin{array}{c}\text { Channel } \\
\text { package }\end{array}$ & CP1 & CP2 & CP3 \\
\hline $\begin{array}{c}\text { IPTV } \\
\text { channels }\end{array}$ & $\begin{array}{c}\text { Basic } \\
\text { channels }\end{array}$ & $\begin{array}{c}\text { Premium } \\
\text { channels }\end{array}$ & $\begin{array}{c}\text { Special } \\
\text { channels }\end{array}$ \\
\hline VLAN & VID1 & VID2 & VID3 \\
\hline LGID & LGID1 & LGID2 & LGID3 \\
\hline ONU & ONU1 & ONU2 & ONU3 \\
& ONU2 & ONU3 & \\
\hline
\end{tabular}

(b) IPTV channel table

\begin{tabular}{c|c|c}
\hline Channel ID & $\begin{array}{c}\text { IP multicast } \\
\text { address }\end{array}$ & $\begin{array}{c}\text { Multicast MAC } \\
\text { address }\end{array}$ \\
\hline
\end{tabular}

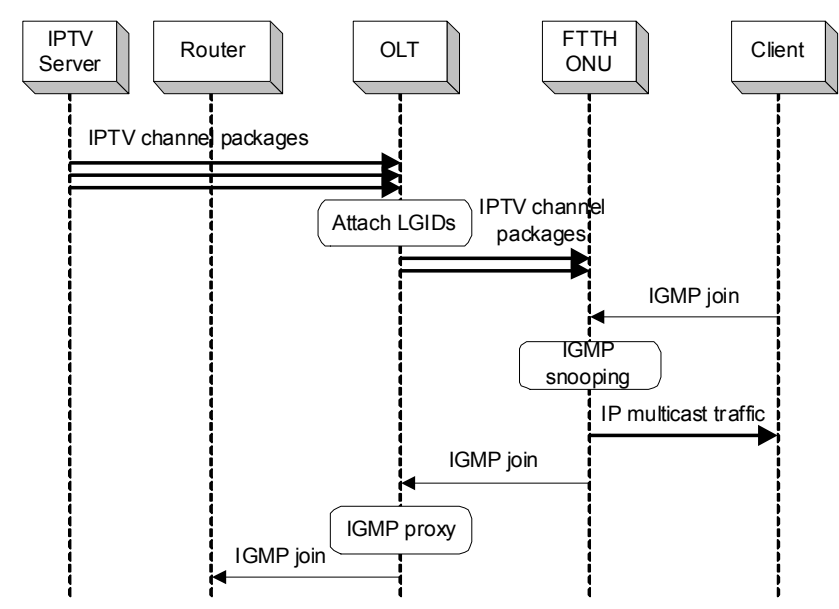

FIG. 8. IPTV channel package delivery using LGID

scribe to certain conditional access (CA) channels or channel packages. If subscription, authorization, and billing are done per ONU, the permission for each subscriber to view specific channels can be accomplished based on registered ONUs. Using the proposed multiple shared LAN emulation, the OLT can provide the ONU with differentiated IPTV channel packages. Also, IP multicast groups are used to convey each IPTV channel from the ONU to the subscriber. This does not require additional management overhead, and the channel switching information just travels to the ONU.

We assume that the IPTV channels consist of a basic channel package $(\mathrm{CP} 1)$, premium channel package (CP2), and special channel package (CP3). An example of the management of differentiated IPTV channel packages is shown in Table 2. CP1 is assigned the VLAN and LGID values, VID1 and LGID1, respectively. These channels are delivered to all ONUs. CP2 is as- signed the VLAN and LGID values, VID2 and LGID2, respectively. Premium channels are delivered to ONU2 and ONU3. When special channels are subscribed to by ONU3, these channels are only delivered to this ONU.

The IPTV channel package delivery procedure is shown in Figure 8. We assume that the IPTV channel packages are delivered to the OLT. The OLT assigns a predefined LGID value to each channel package and delivers them to the ONUs. Each ONU accepts only the allowed channel packages by checking the LGID value of the Ethernet frame. When the user sends the IGMP request to the ONU, the ONU performs IGMP snooping and forwards the requested channel to the user. The ONU forwards the IGMP request to the OLT which then starts up IGMP proxy to judge whether to forward the request to the multicast router or not. When the OLT forwards the IGMP request, we assume that a new channel package which contains the requested IPTV channel is delivered to the OLT. The OLT maintains a mapping table between the multicast address and ONUs, which can be used to tell the OLT which ONUs the multicast stream should be forwarded to. Using the LGID, the VLAN protocol is used for the multicast of the IPTV channel package between the OLT and ONUs, and the channel delivery is performed by the IGMP snooping mechanism.

Service quality requires adequate capacity. To ensure IPTV services, EPONs must provide sufficient bandwidth to ensure delivery without too much loss or delay. 10G EPONs, which offer a significant increase in performance over $1 \mathrm{G}$ EPONs, can provide the increased bandwidth needed by the emerging bandwidthintensive services. IPTV channel delivery using LGID, which is shown in Fig. 7, is suitable for 1G EONs. In this case, IPTV channel packages are delivered to the OLT and the requested channels are delivered from the OLT to the ONUs. As shown in Fig. 8, when 10G EPONs with LGID are used for IPTV distribution, the differentiated IPTV channel packages are delivered from the OLT to each ONU by ONU-based VLAN, and the ONU delivers the requested channels to the STB using the IGMP snooping mechanism.

\section{Channel Zapping Delay}

End users should be able to select the program they wish to view from the available TV contents. This also requires short channel selection and channel changing times. Hence, the channel zapping delay is one of the key IPTV features needed for successful deployment [12]. The overall channel zapping process is shown in Figure 9 [13]. EPONs can be deployed between the home gateway (HG) and gateway router (GWR). Another method of deploying EPONs is to make the FTTH ONU play the role of the HG. As the quality of experience (QoE) parameter, the channel zapping time $\left(\mathrm{T}_{z}\right)$ can be used, which is made up of three 


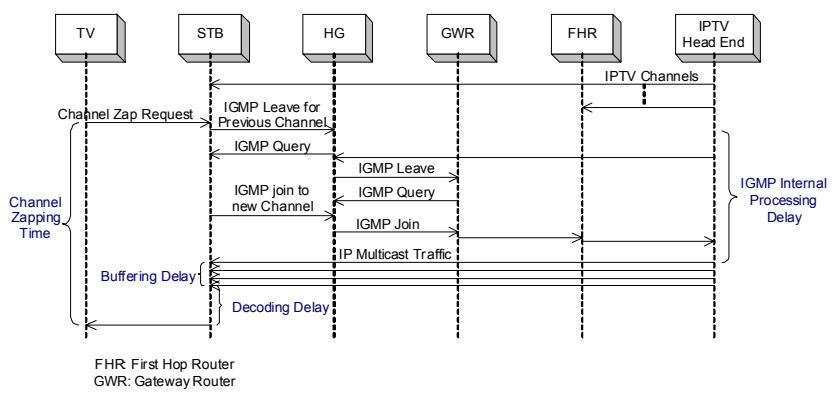

FIG. 9. Overall channel zapping process.

components: the IGMP processing time or network layer zap time $\left(T_{n}\right)$, buffering time $\left(T_{b}\right)$, and decoding time $\left(\mathrm{T}_{\mathrm{d}}\right)$. The IGMP processing time or network layer zap time is the time interval between the transmission of a channel leave message and the reception of the first multicast packet of the requested channel. A channel zap request is triggered by a channel change which is mapped by STB to a multicast group address carried in the IGMP message. After an IGMP message is sent toward a rendezvous point by a gateway router (GWR), the corresponding channel data is delivered to the STB. During the time the STB receives the IPTV multicast traffic, it stacks the packets in a buffer. The buffering delay is the time between the arrival of the first multicast traffic in the buffer and the time when the buffer is sufficiently full to start displaying the video on the screen. After the STB starts to receive and buffer the multicast stream, there is a decoding delay before the buffered data is actually rendered on the TV screen. This type of delay is caused by two processes, codec decoding, whose purpose is to extract program-specific information frames in order to decide the target channel, and I-frame acquisition, which is for the reduction of the bandwidth required for digital video transmission.

To ensure interactivity and satisfactory QoE, the channel zapping delay needs to be below 2 seconds [14]. Typical STB de-jitter buffers can store 100 $500 \mathrm{~ms}$ of video, so network jitter must be within these limits. Generally, IGMP processing takes less than $100 \mathrm{~ms}$, and most of this delay is made up of the buffering delay, because in the ordinary case the decoding delay is less than $100 \mathrm{~ms}$. Moreover, the buffering and decoding delays have a trade-off relationship, the greater the amount of buffer data, the faster the decoding process.

To reduce the channel zapping delay, we only consider the network layer zapping time, because the buffering time and decoding time are beyond the scope of this paper. When the user changes the current channel, IP STB generates an IGMP leave message for the current channel and sends it to the home gateway. After receiving the IGMP leave message, the home gateway sends an IGMP group-specific query message to the home network, in order to see if any host has membership to the group specified in the leave message. If no reports are received after the response time of the last query expires, the home gateway assumes that the group has no local members, stops the forwarding of the multicast stream corresponding to the group, and sends its upper router an IGMP leave message for the group. After the IP STB sends the IGMP leave message to the home gateway, it sends an IGMP join message for the new channel. The home gateway receives this message and sends an IGMP join message to the upper router if no other local hosts have membership for the group. GWR receives the join message and sends a protocol independent multicast (PIM) join message to the other multicast routers in the access network. Then, the multicast stream for the group corresponding to the new channel can be transmitted to the IP STB. In thissituation, the network layer channel zapping time $\left(\mathrm{T}_{\mathrm{n}}\right)$ should be the sum of the IGMP leave processing time $\left(\mathrm{T}_{\text {leave }}\right)$, IGMP join processing time $\left(T_{\text {join }}\right)$, and multicast stream forwarding time to the IP STB ( $\mathrm{T}_{\text {forward }}$ ):

$$
\begin{aligned}
& T_{z}=T_{n}+T_{b}+T_{d} \\
& T_{z}=T_{n}+T_{b}+T_{d}
\end{aligned}
$$

The traditional IPTV service model incurs a network layer channel changing delay, since the channel changing information has to travel upstream through the network to the service provider. If IPTV channel packages were delivered to the OLT using a high-capacity backplane and differentiated IPTV channel packages were delivered to the ONU, the distance the IGMP messages need to travel to effect channel switching would be greatly reduced, thereby providing a shorter channel zapping time.

\section{Simulations}

We assume that 16 ONUs are connected to an OLT. The OLT delivers the requested IPTV channels or channel packages to each ONU. The network traffic model is characterized by self-similarity and long-range dependence (LRD). The frame delays of the upstream and downstream traffic of the ONUs are analyzed for the purpose of estimating the network layer channel zapping time.

In the upstream traffic simulation, the cyclic pollingbased dynamic bandwidth allocation (DBA) algorithm with strict priority scheduling (SPR) is used [6]. The cyclic polling time is $1 \mathrm{~ms}$. For the high-priority service, the emulation of a T1 connection, which consumes a bandwidth of $4.48 \mathrm{Mbit} / \mathrm{s}$, and IGMP control frames are considered. For the medium-priority service, VBR video streams that exhibit the properties of self-similarity 


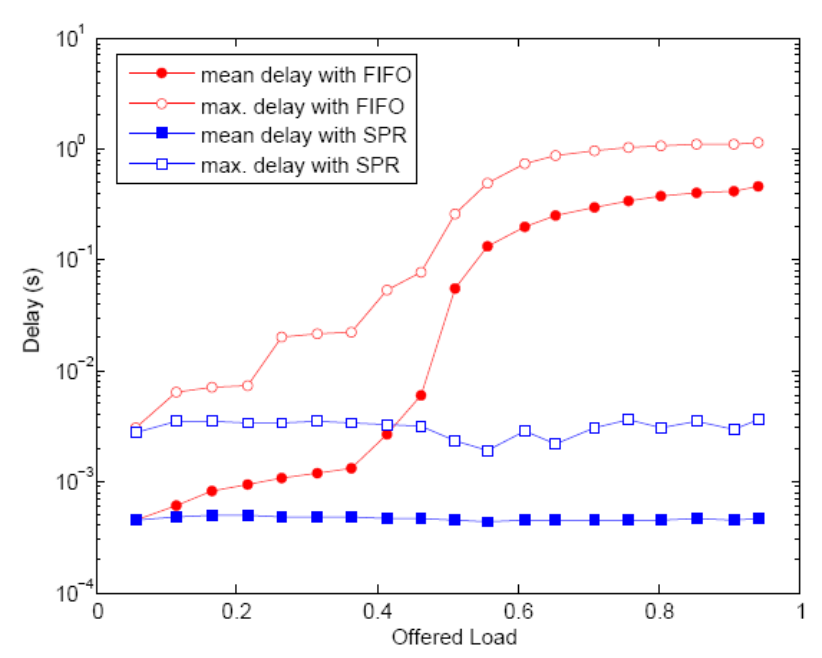

FIG. 10. IGMP control frame delays of upstream traffic.

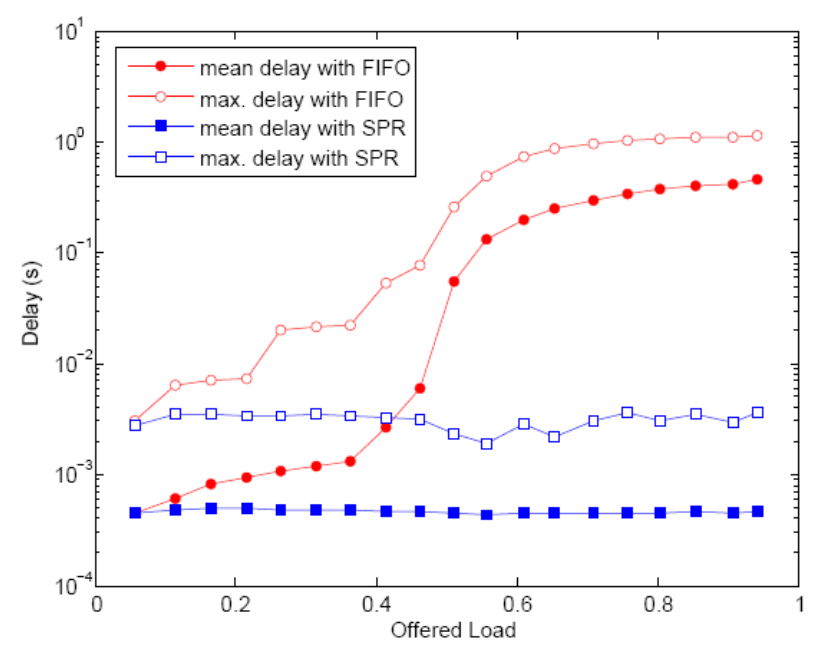

FIG. 11. Frame delay of IPTV traffic and network layer channel zapping time.

and LRD are considered. For the low-priority service, non-real-time data transfer is considered. When the load in the simulation is varied, that $(5 \mathrm{Mbit} / \mathrm{s})$ of the high-priority service is kept constant and the remaining load is split equally between the medium-priority and low-priority services. In the performance diagrams, the ONU's offered load values are normalized to the ONU's ingress link capacity (100 Mbit/s).

Figure 10 shows the IGMP control frame delays of the upstream traffic of the ONUs. As the load increases from light to heavy, the mean and maximum delays are increased when the FIFO queue processing technique is used. When the cyclic polling-based DBA with SPR is applied, the mean frame delay of the IGMP control messages is about $0.5 \mathrm{~ms}$ with various offered ONU loads. The maximum frame delay also remains at about $3 \mathrm{~ms}$ with various offered ONU loads. Hence, the DBA algorithm with SPR is adequate for the transmission of the upstream traffic.

In the downstream traffic simulation, the high-priority service is considered to have a constant load (5 Mbit/s) for each ONU, as in the case of the upstream traffic simulation. For the medium-priority service, IPTV channels that require roughly $9 \mathrm{Mbit} / \mathrm{s}$ for each high definition TV (HDTV) channel using advanced video compression are considered. For the low-priority service, best effort traffic $(15 \mathrm{Mbit} / \mathrm{s})$ is considered for each ONU. We vary the load of the medium-priority traffic by increasing the number of HDTV channels delivered to the STB.

Figure 11 shows the IPTV frame delays of the ONUs and network layer channel zapping delays in the case where IPTV channel delivery with LGID is applied. The network layer channel zapping time $\left(T_{n}\right)$ is obtained as the sum of the upstream IGMP frame delays of the ONUs, the round-trip time (RTT), the downstream IPTV channel delay of the ONUs, and the additional OLT or/and ONU processing time $(1 \mathrm{~ms})$. As the number of IPTV channels increases, the frame delays and network layer channel zapping times are increased. When the load of the IPTV traffic of the ONU is below the ONU's link capacity (100 Mbit/s), the mean and maximum network layer channel zapping times are about $2 \mathrm{~ms}$ and $5 \mathrm{~ms}$, respectively.

When IPTV channel package delivery with VLAN and IGMP snooping is applied, the network layer channel zapping time $\left(\mathrm{T}_{\mathrm{n}}\right)$ can be considered to be instantaneous, because the RTT and frame delays of the upstream and downstream traffics are not considered. However, the proposed IPTV channel package delivery consumes more downstream bandwidth of the EPON.

\section{CONCLUSIONS}

We propose an IPTV channel delivery model for 10G EPONs. By using logical group identification, ONUbased multicast or multiple shared LAN emulation is proposed for EPONs. Using ONU-based VLAN services, EPONs can support separate and secure connections between providers and subscribers. When 10G EPONs with LGID are used for IPTV distribution, differentiated IPTV channel packages are delivered from the OLT to each ONU, and the ONU delivers the requested channels to the STB. Hence, ONU-based VLAN and the IGMP snooping mechanism enable EPONs to provide differentiated and secure IPTV channel package delivery services, while shortening the channel zapping time.

\section{ACKNOWLEDGEMENT}

This work was supported by the Korea Research Foundation Grant funded by the Korean Government 
(MOEHRD, Basic Research Promotion Fund) (KRF2007-331-D00312).

\section{REFERENCES}

[1] IEEE Standard 802.3ahTM-2004, Ethernet in the First Mile, 2004.

[2] IEEE Standard 802.1D, Media Access Control (MAC) Bridges, 2004.

[3] S. I. Choi and J. D. Huh, "Dynamic Bandwidth Allocation Algorithm for Multimedia Services over Ethernet PONs," ETRI Journal, vol. 24, no. 6, pp. 465468, 2002.

[4] G. Pesavento, B. Mukherjee, S. Dixit, Y. Ye, and R. Hirth, "Supporting Differentiated Classes of Service in Ethernet Passive Optical Networks," Journal of Optical Networking, vol. 1, nos. 8 \& 9, pp. 280-298, 2002.

[5] C. M. Assi, Y. Ye, S. Dixit, and M. A. Ali, "Dynamic Bandwidth Allocation for Quality-of-Service Over Ethernet PONs," IEEE Journal on Selected Areas in Communications, vol. 21, no. 9, pp. 1467-1477, 2003.

[6] S. I. Choi, "Cyclic Polling-Based Dynamic Bandwidth Allocation for Differentiated Classes of Service in Ethernet Passive Optical Networks," Photonic Network Comm., vol. 7, no. 1, pp. 87-96, 2004.

[7] N. Finn, "Two Models for IEEE 802.3ah EPONs," IEEE 802.3ah EFM, Edinburgh, UK, May 2002: URL (http://www.ieee802.org /3/efm/public/may02/finn_3_ 0502.pdf).

[8] K. Kerpez, D. Waring, G. Lapiotis, J. B. Lyles, and R. Vaidyanathan, "IPTV Service Assurance," IEEE Communications Magazine, vol. 44, no. 9, pp. 166-172, 2006.

[9] X. Lu, D. Liu, M. Mao, and J. Wang, "Design and Implementation of Digital Television over Ethernet PON Transmission System," Proc. Of SPIE, vol. 6022, pp. 938-948, 2005.

[10] J. Wu, Y. Nie, H. Yoshiuchi, and H. Ikeda, "Building Multicast Controller for Carrier-grade IPTV Service over Ethernet Passive Optical Network," Proc. of Second International Conference on Systems and Networks Communications (ICSNC 2007), pp. 25-31, 2006.

[11] M. Takizawa and T. Yokomoto, "Multicast Logical Link for 10G-EPON," Presentation on 10Gb/s PHY for EPON Study Group, IEEE802.3 Interim Meeting, 2006.

[12] S. Vedantham S.-H. Kim, and D. Kataria, "GarrierGrade Ethernet Challenges for IPTV Deployment," IEEE Communications Magazine, vol. 44, no. 7, pp. 24-31, 2006.

[13] J.-H. Bae, H.-S. Park, and J.-H. Hahm, "Consideration on channel zapping time in IPTV performance monitoring," ITU-T Focus Group on IPTV, 4th FG IPTV Meeting, 2007.

[14] DSL Forum TR-126, Triple-play Services Quality of Experience Requirements, 2006. 